\title{
Salivary biomarkers and proteomics: future diagnostic and clinical utilities
}

\author{
Biomarkers e proteomica salivari: prospettive future cliniche e diagnostiche \\ M. CASTAGNOLA 1 , E. SCARANO², G.C. PASSALI², I. MESSANA ${ }^{3}$, T. CABRAS ${ }^{4}$, F. IAVARONE ${ }^{5}$, G. DI CINTIO², \\ A. FIORITA2 ${ }^{2}$, E. DE CORSO2 ${ }^{2}$ G. PALUDETTI2 \\ ${ }^{1}$ Institute of Biochemistry and Clinical Biochemistry, Catholic University, Rome, Istituto di Chimica del \\ Riconoscimento Molecolare C.N.R. Rome, Italy; ${ }^{2}$ Department of Head and Neck Surgery, "A. Gemelli" Hospital \\ Foundation, Catholic University, Rome, Italy; ${ }^{3}$ Life and Enviromental Sciences Department, University of Cagliari, \\ and Istituto di Chimica del Riconoscimento Molecolare C.N.R. Rome, Italy; ${ }^{4}$ Life and Enviromental Sciences \\ Department, University of Cagliari, Italy; ${ }^{5}$ Institute of Biochemistry and Clinical Biochemistry, Catholic University, \\ Rome, Italy
}

\begin{abstract}
SUMMARY
Saliva testing is a non-invasive and inexpensive test that can serve as a source of information useful for diagnosis of disease. As we enter the era of genomic technologies and -omic research, collection of saliva has increased. Recent proteomic platforms have analysed the human salivary proteome and characterised about 3000 differentially expressed proteins and peptides: in saliva, more than $90 \%$ of proteins in weight are derived from the secretion of three couples of "major" glands; all the other components are derived from minor glands, gingival crevicular fluid, mucosal exudates and oral microflora. The most common aim of proteomic analysis is to discriminate between physiological and pathological conditions. A proteomic protocol to analyze the whole saliva proteome is not currently available. It is possible distinguish two type of proteomic platforms: top-down proteomics investigates intact naturally-occurring structure of a protein under examination; bottom-up proteomics analyses peptide fragments after pre-digestion (typically with trypsin). Because of this heterogeneity, many different biomarkers may be proposed for the same pathology. The salivary proteome has been characterised in several diseases: oral squamous cell carcinoma and oral leukoplakia, chronic graft-versus-host disease Sjögren's syndrome and other autoimmune disorders such as SAPHO, schizophrenia and bipolar disorder, and genetic diseases like Down's Syndrome and Wilson disease. The results of research reported herein suggest that in the near future human saliva will be a relevant diagnostic fluid for clinical diagnosis and prognosis.
\end{abstract}

KEY WORDS: Saliva $\bullet$ Proteome $\bullet$ Salivary biomarkers

\section{RIASSUNTO}

Lo studio della proteomica salivare, test economico e non invasivo, rappresenta una fonte di numerose informazioni, ed è utile per la diagnosi di svariate malattie. Da quando siamo entrati nell'era della tecnologia genomica e delle scienze "omiche", la raccolta di campioni salivari è aumentata esponenzialmente. Recenti piattaforme proteomiche hanno analizzato il proteoma salivare umano, caratterizzando circa 3000 peptidi e proteine, espressi in maniera differente: più del 90\% in peso deriva dalla secrezione delle tre ghiandole salivari maggiori, mentre la restante parte proviene dalle ghiandole salivari minori, dal fluido crevicolare gengivale, da essudati mucosi e dalla microflora orale. L'obiettivo principale dell'analisi proteomica è discriminare tra condizioni fisiologiche e patologiche. Ad oggi, tuttavia, non esiste un preciso protocollo che permetta di analizzare l'intero proteoma salivare, pertanto sono state realizzate svariate strategie. Innanzitutto, è possibile distinguere due tipologie di piattaforme proteomiche: l'approccio "top-down" prevede l'analisi delle proteine sotto esame come entità intatte; nell'approccio "bottom-up" la caratterizzazione della proteina avviene mediante lo studio dei peptidi ottenuti dopo digestione enzimatica (con tripsina tipicamente). A causa di questa eterogeneità, per una stessa patologia sono stati proposti differenti biomarkers. Il proteoma salivare è stato caratterizzato in numerose malattie: carcinoma squamoso e leucoplachie orali, malattia del trapianto contro l'ospite (GVHD) cronica, sindrome di Sjögren e altri disordini autoimmuni come la sindrome SAPHO (sinovite, acne, pustolosi, iperostosi e osteite), schizofrenia e disordine bipolare, malattie genetiche come la sindrome di Down o la malattia di Wilson. In conclusione, i risultati delle ricerche riportate in questa review suggeriscono che nel prossimo futuro la saliva diverrà un fluido di indubbia rilevanza diagnostica utile per fini clinici, sia diagnostici, sia prognostici.

PAROLE CHIAVE: Saliva $\bullet$ Proteoma $\bullet$ Biomarkers salivari

Acta Otorhinolaryngol Ital 2017;37:94-101

\section{Introduction}

Saliva is a very attractive body fluid for diagnosis of disease for many reasons: i) collection of saliva is usually economical, safe, easy and can be performed without the help of healthcare workers (it allows for home-based sam- pling); ii) it is considered an acceptable and non-invasive process by patients because it does not provoke any pain (and can be easily collected for patients in the paediatric age range) ${ }^{1}$. Recent proteomic platforms have analysed the human salivary proteome, characterising about 3000 
differentially expressed proteins and peptides, many of them of microbiological origin ${ }^{2}$. A careful evaluation of this huge amount of data so far achievable will allow, in the near future, to tailor therapeutic interventions by assessment of thousands of parameters. Today, proteomic technologies are extremely complex, expensive and of limited accessibility. It is, however, not difficult to foresee an explosion in -omics research applications in the next years, with production of simple, inexpensive and ergonomic instruments, that can be applied to small salivary samples for early diagnosis of different pathologies. The aim of this review is to briefly describe the most salient aspects of current proteomic researches and other-omic sciences carried out on human saliva with particular regard to its potential use as a diagnostic fluid and to underline the most demanding and challenging perspectives.

\section{The human saliva proteome}

As with any bodily fluid, whole human saliva has specific characteristics, and some recent reviews have described the distinctiveness of its proteome ${ }^{34}$. More than $90 \%$ in weight of the about 3,000 protein components detected in saliva ${ }^{5}$ are derived from the secretion of three couples of "major" glands, parotid, sub-mandibular and sub-lingual ( $\mathrm{Sm}-\mathrm{Sl})$ glands, and pertain to the classes of proline-rich proteins (PRPs; divided in acidic, basic and basic glycosylated), $\alpha$-amylases, mucins, salivary ("S-type") cystatins, histatins, statherin and P-B peptide. All these components and derivatives account for about 200 proteins/ peptides. All the other components detected in saliva represent the remaining $10 \%$ in weight. Some of these, i.e. lipocalin, are secreted by minor glands (labial, palatine, buccal and lingual, i.e. von Ebner glands) ${ }^{5}$, Others, such as $\alpha$-defensins and $\beta$-thymosins, derive mainly from gingival crevicular fluid ${ }^{67}$. Human serum albumin and other plasmatic proteins are probably the products of mucosal exudates, while others are of exogenous (oral microflora) origin.

Major families of secreted salivary proteins are polymorphic, and various post-translational modifications (PTMs) occur before secretion, such as glycosylation, phosphorylation, exo- and endo-proteolytic cleavages, as reported in recent reviews ${ }^{138}$. A small percentage of histatin 1 is submitted to tyrosine sulphation ${ }^{9}$. Cystatin B is detectable mainly as S-glutathionyl and S-cysteinyl derivatives ${ }^{10}$. The most common aim of proteomic analysis is to discriminate between physiological and pathological conditions. In the presence of multiple sources, such as in the case of salivary glands, quantitative alteration of one source might be compensated by others. The composition of whole saliva varies depending on different physiological conditions. Minor glands secrete during the night spontaneously at a low rate. In daytime and at rest, movements of the tongue and lips, and mucosal dryness stimu- late secretion, particularly by the submandibular gland (unstimulated secretion). In response to strong stimuli, parotid contributions become more dominant, with a flow-rate about twice as high as that from the submandibular gland when chewing. On the whole, the flow rate of resting as well as stimulated saliva is higher in the afternoon than in the morning, the peak occurring in the middle of the afternoon. Age is another important variable affecting the salivary proteome. Indeed, recent studies have indicated that secretion of specific peptides is noticeably different in the paediatric age with respect to adults ${ }^{11} 12$. This dynamism is challenging for proteomic investigations of human saliva ${ }^{3}$ and all sources of variability must be carefully considered for choice of the proper control group. Nonetheless, because many PTMs occurring during glandular secretion are under the action of enzymes common to other exocrine and endocrine glands, qualitative and quantitative alterations may be a clue of parallel malfunctions of other exocrine and endocrine glands, and therefore a signal of systemic diseases.

\section{Proteomic platforms for the study of human saliva}

Because a proteomic strategy able to characterise the whole saliva proteome does not exist ${ }^{12}$ and many studies on the same disease have been carried out with different instruments and experimental plans, it is not surprising that different biomarkers have been proposed for the same pathology. However, different biomarkers are sometimes reported when applying similar platforms to the same pathology, generating legitimate doubts on the robustness of the experimental plan utilised, on the number of samples under study and on the choice of proper controls ${ }^{13}$. These aspects were nicely outlined in several studies ${ }^{14} 15$ showing that the increased number of components under observation strongly enhances the possibility to detect variations connected to inter-individual polymorphisms. With the exception of Sjögren's syndrome, several studies carried out to detect biomarkers in the same disease have often produced inconsistent results. It is advised that an adequate number of samples are analysed that can provide highly significant statistical differences, to strictly follow identical experimental protocols for different groups of samples and to analyze them in random order. The use of ELISA methods for validation of proteomic results has also been debated ${ }^{12}$, because the antibody utilised may not have the proper selectivity to discriminate between the proteoforms connecterelated with development of the disease.

Different classifications are available for proteomic platforms. Depending on the sample, they are first divided into bottom-up and top-down platforms. Top-down proteomics investigates the intact naturally occurring structure of a protein under examination, avoiding as much as 
possible any sample alterations. Bottom-up proteomics is centered on pre-digestion of the sample (typically with trypsin) followed by the analysis of peptide fragments by high-throughput analytical methods. The presence of a protein in the sample is inferred by the detection of one or more of its specific (proteotypic) fragments, implying biunivocal correspondence between the parent protein and its fragments ${ }^{12}$. The majority of proteins are submitted to extensive post-translational modifications, cleavages included, before reaching a mature functional structure. As a consequence, the minimalistic approach of the bottomup strategy can result in the relevant loss of important molecular information. PTMs are difficult highlight in bottom-up shotgun experiments, where the vast majority of peptide sequences are often associated with a specific cDNA sequence, thus leveling out at a statistical level the presence of a PTM. Moreover, the association of molecular maturation events associated with the specific onset of a defined PTM will not be directly accessible by bottom-up shot-gun experiments. This defect is relevant for the proteome of human saliva, where many proteins, i.e. basic and acidic PRPs, are not very susceptible to the action of proteolytic enzymes and disclose very similar sequences. Thereby, many fragments cannot be related to a specific parent protein. Nonetheless, bottom-up platforms have shown the best throughput in terms of number of detected components. The sensitivity of new generation mass spectrometers is enough to reveal thousands of peptides in a single analysis, while the main problem is related to the increase in time necessary for the different separation steps ${ }^{16}$. Therefore, improvements in the separation platform utilised in shot-gun proteomics reflect in easier peptide detection. In this way, shot-gun proteomics covers the highest range of detectable components, regardless of their mass, because the proteolytic digestion of large proteins can almost always generate proteotypic peptides that can disclose the presence of the parent protein in a complex mixture. For these reasons, the number of salivary components currently detectable by shot-gun approaches is more than five times greater than the number of components detected by any other platform. Top-down platforms are intrinsically limited by the sample treatments necessary for coupling with mass spectrometry (typically treatment with formic acid or trifluoroacetic acid), which inevitably excludes proteins that are insoluble in acidic solution. Moreover, intact high-molecular weight proteins and heterogeneous glycosylated proteins are not accessible, in their naturally occurring forms, even to the best high-level MS apparatus. Platforms based on 2-D-electrophoresis are affected by poor reproducibility and to avoid bias it is often necessary to run multiple replica of the same sample. The results obtainable by MALDI-TOF-MS are strongly dependent on the formation of the matrix layer. Therefore, any proteomic platform has advantages and drawbacks. For all the above reported reasons, the best way to carry out a robust biomarker identification is to analyse an adequate number of samples with different proteomic methodologies, even though this possibility is not accessible to the majority of laboratories ${ }^{12} 15$.

\section{Human saliva as diagnostic body fluid}

Several excellent reviews have recently been published outlining the possibility to use saliva as a diagnostic fluid $^{17-19}$. As a consequence, we will report only some of the most recent research carried out in the last three years, apologising for relevant omissions. More than $90 \%$ of oral cancers are oral squamous cell carcinoma (OSCC). Many patients are diagnosed with the tumour at a late state with poor prognosis and low survival rate; early diagnosis of OSCC is thus urgent problem for clinicians. Many recent proteomic studies have been devoted to the search for early salivary biomarkers of OSCC and other oral cancers. The results obtained add further information to numerous previous studies on this topic, which have suggested 17 up-regulated protein biomarkers ${ }^{17}$. Among these, interleukins 6,8 and $1 \beta$, cyclin D1 thioredoxin and profiling 1 seem to be the most promising. The proteome of saliva from three groups of patients (healthy controls, individuals with potentially malignant disorders (OPMD) and OSCC patients) was investigated by SDS-PAGE coupled to LC-MS/MS. In the control, OPMD and OSCC groups 958, 845 and 1030 salivary proteins were detected, respectively. By label free quantification, 22 overexpressed proteins were detected in the OSCC group. Among these, resistin (RETN) was validated by ELISA thus confirming proteomic data. RETN levels had significant correlation with late-stage primary tumours, advanced overall stage and lymphnode metastasis ${ }^{20}$. The same group used a spectral counting-based label free quantification platform to identify 64 protein candidates for OSCC ${ }^{21}$. Retrieving mRNA expression from public-domain based transcriptome data sets, they were able to reduce the number of potential candidates to 19 . Among these, thrombospondin-2 was identified as the best biomarker because higher levels were associated with a higher overall pathological state, positive perineural invasion and poorer prognosis ${ }^{21}$. Using nano-LC-MS/MS and validation by Western blot and ELISA, Jou et al. ${ }^{22}$ were able to identify S100A8 as a potential biomarker of OSCC. High level of S100A8 appeared in 3.4, 13.9, 92.9 and $100 \%$ of saliva of OSCC patients with T1, T2, T3 and T4 stages, respectively. The AUROC curve indicated high sensitivity, specificity and accuracy of S100based ELISA as a detector. A comparative 2-D electrophoretic analysis of whole saliva of patients with OSCC $(\mathrm{n}=12)$ and healthy controls $(\mathrm{n}=$ 12) was able to identify $\alpha 1$-antitrypsin (AAT), haptoglobin $\beta$ chains (HAP), complement $\mathrm{C} 3$, haemopexin and transthyretin as potential OSCC biomarkers, which were 
validated by ELISA. In particular, a strong association of ATT and HAP with OSCC was further supported by immunochemical staining of cancer tissues ${ }^{23}$. A targeted proteomic strategy applying a MS selected reaction monitoring (SRM) assay to 14 OSCC candidate biomarker proteins suggested that AAT, complement $\mathrm{C} 3,4 \mathrm{~B}$, factor $\mathrm{B}$, and leucine-rich $\alpha$-2-glycoprotein are associated with increased risk to develop OSCC ${ }^{24}$.

Using an affinity-based depletion method to eliminate amylase and albumin coupled to high-resolution $\mathrm{MS}, \mathrm{Si}-$ vadasan et al. ${ }^{25}$ were able to identify 1256 salivary proteins and to update the salivary proteome to 3449 proteins, 806 of which were differentially expressed in oral cancer tissues ${ }^{25}$. The authors provide a list of 139 proteins along with their proteotypic peptides, which might serve as a reference for targeted investigations as secretory markers for clinical applications in oral malignancies. A study carried out with a 2D-PAGE platform and Western blot validation identified (among 880 spots, corresponding to 151 different gene products) galectine-7 as a good salivary biomarker for OSCC, with a specificity of $90 \%$ and a sensitivity of $80.5 \%(n=10){ }^{26}$. The search for early biomarkers of OSCC was also carried out with transcriptomic and metabolomic platforms and some articles have reviewed these topics ${ }^{18} 27$. A metabolomic study carried out with uHPLC coupled to Q-TOF MS on whole saliva from 37 OSCC patients, 32 patients with oral leukoplakia (OLK) and 34 healthy subjects showed characteristic metabolic signatures for the three groups. A panel of five metabolites (phenylalanione, valine, n-eicosanoid acid, lactic acid and $\gamma$-aminobutyric acid) was selected by statistical methods. After evaluation of the predictive power of the five metabolites, the authors established that valine, lactic acid and phenylalanine in combination yielded satisfactory accuracy $(0.89$ and 0.97$)$, sensitivity $(86.5 \%$ and $94.6 \%)$, specificity $(82.4 \%$ and $84.4 \%$ ) and positive predictive value $(81.6 \%$ and $87.5 \%)$ in distinguishing OSCC from controls and OLK, respectively ${ }^{28}$. A similar metabolomic study carried out with hydrophilic interaction chromatography (HILIC) coupled to TOF-MS on whole saliva of OSCC patients identified five potential biomarkers: propionylcholine, $\mathrm{N}$-acetyl-L-phenylalanine, sphinganine, phytosphingosine and S-carboxymethyl-L-cysteine. Their combination yielded satisfactory accuracy $(0.977)$, sensitivity (100\%) and specificity (96.7\%) in distinguishing early stage of OSCC from controls ${ }^{29}$.

Recent research has suggested that potential biomarkers in other cancer types may be present in human saliva. A study carried out by nano-HPLC-Q-TOF MS investigated the proteome profiles of plasma and saliva of patients with fibroadenoma $(\mathrm{n}=10)$, infiltrating ductal carcinoma $(\mathrm{n}=10)$ and healthy controls $(\mathrm{n}=8)$. The major differentially expressed proteins in the saliva of patients compared with controls were $\alpha 2$-macroglobulin and ceruloplasmin, which should be further validated as potential biomarkers of impalpable breast lesions ${ }^{30}$. A differential proteomic analysis using tandem mass tags technology was performed to characterise potential salivary biomarkers for gastric cancer detection. More than 500 proteins were identified and quantified, and three were successfully verified by ELISA, namely cystatin $B$, triose-phosphate isomerase and a protein called "deleted in malignant tumor 1 protein". The combination of these three biomarkers could reach $85 \%$ sensitivity and $80 \%$ specificity for the detection of gastric cancer with accuracy of $0.93{ }^{31}$. A 2D-electrophoretic analysis coupled off-line with MS identification of the tryptic digest of the spots identified 22 proteins selectively expressed in patients with oral leukoplakia ${ }^{32}$. Immunohistochemical validation suggested that keratin 10 was an interesting potential biomarker of OLK and should be further investigated. A transcriptomic platform identified five mRNA biomarkers (CCNI, EGFR, FGF19, FRS2 and GREB1) that after logistic regression model can differentiate lung cancer patients from normal subjects ${ }^{33}$.

The proteome of saliva seems to have the potential to discriminate many other diseases. Two groups have investigated whole human saliva to find potential signatures in oral chronic graft-versus-host-disease (cGVHD), a severe immunological complication occurring after allogeneic haematopoietic stem cell transplantation ${ }^{3435}$. A LC-MS/ MS study observed a reduction of salivary lactoperoxidase, lactotransferrin and several proteins included in the cysteine proteinase inhibitor family suggesting impaired oral antimicrobial host immunity in cGVHD patients ${ }^{34}$. Another study performed utilising iTRAQ labeling followed by HPLC-ESI-MS/MS and ELISA validation showed decreased expression of IL-1 receptor antagonist and cystatin $\mathrm{B}$ in saliva of patients with active oral cGVHD. ROC analysis revealed that these two markers were able to distinguish oral cGVHD with a sensitivity of $85 \%$ and specificity of $60 \%{ }^{35}$

Many proteomic studies in the past were devoted to the characterisation of salivary biomarkers of Sjögren's syndrome (pSS) and this topic has been reviewed in depth ${ }^{36-38}$. A recent study investigated the expression of thymosins $\beta 4$ and $\beta 10$ in patients with pSS and in patients with autoimmune diseases: systemic sclerosis [SSc], systemic lupus erythematosus [SLE] and rheumatoid arthritis [RA], with and without sicca syndrome [ss]. This research showed that higher salivary $\mathrm{T} \beta$ expression characterised patients with $\mathrm{pSS}$, while $\mathrm{T} \beta_{4}$ sulfoxide and $\mathrm{T} \beta_{10}$ salivary expression were selectively present in patients with sicca symptoms, suggesting a different role for $T \beta_{4}$ and $T \beta_{10}$ in patients with pSS who have ss and other autoimmune disease ${ }^{39}$. A metabolomic analysis of saliva from patients with pSS carried out with a GC-MS platform was able to detect a total of 88 metabolites, 41 of which were observed at reduced levels in samples from pSS patients. The reduced presence of glycine, tyrosine, uric acid and 
fucose observed might reflect salivary gland destruction due to chronic sialoadenitis ${ }^{40}$. A top-down HPLC-ESIMS and MS/MS platform was able to detect a signature in whole saliva of patients with synovitis, acne, pustulosis, hyperostosis and osteitis (SAPHO) syndrome, another rare, often unrecognised, rheumatological disease with prominent inflammatory cutaneous and articular symptoms characterised by musculoskeletal manifestations (synovitis, hyperostosis, osteomyelitis) associated with dermatological conditions (severe acne and pustulosis). The acidic soluble fraction of whole saliva from 10 adult women affected by SAPHO syndrome and from a group of 28 healthy women was analysed by RP-HPLC-ESIMS and showed a significantly decreased concentration of cystatin S1 and SN, histatins, the major acidic PRPs, P-C and P-B peptides in saliva of SAPHO subjects with respect to controls. Histatins showed positive correlations with $\mathrm{C}$ reactive protein, cystatin $\mathrm{S} 1$, histatins 3 , histatin 5 and a positive correlation with the neutrophil count, while histatin 3 correlated positively with total white cell count and negatively with the erythrocyte sedimentation rate. The levels and frequency of S100A12 protein showed a trend to increase in SAPHO patients, which was probably related to the inflammatory response and to the altered neutrophil responses to functional stimuli that characterize SAPHO syndrome, suggesting a possible application as a salivary biomarker ${ }^{41}$.

Proteomics of saliva can contribute to the detection of early markers of psychiatric diseases ${ }^{42}$. A recent study carried out with a top-down HPLC-ESI-MS and MS/MS platform investigated whole saliva of 32 subjects with diagnosis of schizophrenia (SZ), 17 with diagnosis of bipolar disorder (BD) and 31 healthy subjects divided in non-smokers (HN; $\mathrm{n}=19)$ and smokers (HS; $\mathrm{n}=12$ ) Both $\mathrm{SZ}$ and $\mathrm{BD}$ revealed more than 10 fold mean increase of $\alpha$-defensins 1-4, S100A12, cystatin A and S-derivatives of cystatin B levels with respect to the HN and HS control groups. This study confirmed schizophrenia-associated dysregulation of a immune pathway of peripheral white blood cells and suggested that the dysregulation in the $\mathrm{BD}$ group could involve the activation of more specific cell type than that of SZ group ${ }^{43}$. A proteomic analysis of saliva in HIV-positive heroin addicts performed by a longitudinal HPLC-MS based quantitative platform investigated saliva samples taken from 8 HIV-positive (HIV+) and 11 -negative (HIV-) heroin addicts. In addition, saliva samples were investigated from $11 \mathrm{HIV}$ - non-heroin addicted healthy controls. In the HIV+ group, 58 proteins were identified that show significant correlations with cognitive scores, implicating disruption of protein quality control pathways by HIV ${ }^{44}$.

Saliva proteome was able to detect signatures that are characteristics of genetic diseases. Whole saliva of 36 Down's syndrome subjects, divided in age groups 10$17 \mathrm{yr}$ and 18-50 yr, was analysed by a top-down prot- eomic approach, and the HPLC-ESI-MS profiles were compared with sex- and age-matched control groups. The main results suggested that levels of the antimicrobial $\alpha$-defensins 1 and 2 and histatins 3 and 5 were significantly increased in whole saliva of older Down's syndrome subjects with respect to controls and that S100A7, S100A8, and S100A12 levels were significantly increased in whole saliva of Down's syndrome subjects in comparison with controls. The increased levels of S100A7 and S100A12 may be of particular interest as a biomarker of early onset of Alzheimer's disease, which is frequently associated with Down's syndrome ${ }^{45}$. A proteomic analysis was carried out by a top-down proteomic platform on whole saliva of Wilson's disease patients. Wilson's disease is a rare inherited disorder of copper metabolism, manifesting hepatic, neurological and psychiatric symptoms. The qualitative/quantitative characterisation of the salivary proteome/peptidome of 32 Wilson's disease patients exhibited significant higher levels of S100A9 and S100A8 proteoforms, and their oxidised forms with respect to controls. Oxidation occurred on methionine and tryptophan residues, and on the unique cysteine residue, in position 42 in S100A8, and 3 in S100A9, that generated glutathionylated, cysteinylated, sulphinic, sulphonic, and disulphide dimeric forms. These findings showed that the salivary proteome of Wilson's disease patients reflected oxidative stress and inflammatory conditions characteristic of the pathology ${ }^{46}$.

A quantitative proteomic analysis based on limited protein separation within the zone of the stacking gel of the 1D SDS-PAGE and using isotope-coded synthetic peptides as internal standards was employed to study the whole saliva proteome of HIV-1 infected individuals. Expression levels of members of the calcium-binding S100 protein family and "deleted in malignant brain tumours 1 protein" were up-regulated, while that of mucin 5B was down-regulated in HIV-1 seropositive saliva samples, suggesting new perspectives for monitoring HIV-infection and understanding the mechanism of HIV-1 infectivity ${ }^{47}$. Since whole saliva contains variegate microflora, several platforms were recently developed to investigate the human salivary metaproteome. A platform combined protein dynamic range compression (DRC), multidimensional peptide fractionation and high-mass accuracy MS/MS with a two-step peptide identification method using a database of human proteins plus those translated from oral microbe genomes was recently studied. Peptides were identified from 124 microbial species. Streptococcus, Rothia, Actinomyces, Prevotella, Neisseria, Veilonella, Lactobacillus, Selenomonas, Pseudomonas, Staphylococcus and Campylobacter were abundant among the 65 genera from 12 phyla represented. Taxonomic diversity was broadly consistent with metagenomic studies of saliva ${ }^{48}$. A bottom-up shotgun nano-HPLC-ESI-MS platform was applied to saliva samples of 10 patients with periodontitis, 10 patients with 
dental caries and 10 orally healthy individuals detecting a total of 35,664 unique peptides from 4,161 different proteins, of which 1,946 and 2,090 were of bacterial and human origin, respectively. The human protein profiles displayed significant overexpression of the complement system and inflammatory markers in periodontitis and dental caries compared to healthy controls, while bacterial proteome profiles and functional annotation were very similar in health and disease. Similar bacterial proteomes in healthy and diseased individuals suggests that the salivary microbiota predominantly thrives in a planktonic state expressing no disease-associated characteristics of metabolic activity ${ }^{49}$.

\section{Conclusions}

Saliva is already used routinely by clinical laboratories for detection of secretory $\operatorname{IgA}$ antibodies, determination of salivary cortisol, hormones and for genetic purposes. However, the results of the research reported in this review suggest that in the near future human saliva will be a relevant diagnostic fluid for clinical diagnosis and prognosis. The application of holistic technologies such as proteomics and other-omic sciences to saliva should soon provide a picture of the incredible complexity of each individual, capturing his/her distinct and unique metabolic fingerprint and the pathways involved in the health/disease state transition and its reverse. Omic sciences are contributing to the identification and characterisation of salivary components, including DNA, RNA, proteins, metabolites and microorganisms. Saliva may contain real-time information describing our overall physiological condition. The -omic studies are showing that, like blood and tissue biopsies, oral fluids can be a source of biochemical data capable of detecting diseases, not only restricted to local disorders like oral cancer and Sjögren's syndrome, but systemic pathologies like genetic, autoimmune, cardiovascular and metabolic diseases as well as viral/bacterial infections and cancers. The main advantage is its easy and non-invasive collection. Moreover, several recent studies are demonstrating that the proteome of whole saliva can be divided in several subproteomes, because this body fluid derives from the contribution of different sources. The future increase of the selectivity, resolution and sensitivity of the proteomic MS-based platforms will allow proteomes deriving from these different sources to be investigated in greater detail. The metaproteomic analyses of human oral microbiota ${ }^{48} 49$ are a nice example of exploitation of subproteomes of saliva for future diagnosis of infectious and opportunistic diseases. Moreover, human saliva contains extracellular vesicles ${ }^{50}$ that can be easily separated and utilised for diagnosis of a large set of diseases with particular regard for cancer. Even though this topic must still be largely explored, a recent study showed the feasibility of the analysis of the subproteome offered by oral vesicles established that it can contribute to early diagnosis and prognosis of OSCC ${ }^{51}$. Another interesting oral subproteome is constituted by gingival crevicular fluid, arising from the gingival plexus of blood vessels in the gingival corium, subjacent to the epithelium lining the dento-gingival space, as it contains a diverse population of cells, including bacteria from the adjacent plaque mass, transmigrating leucocytes and desquamated epithelial cells, which are passively washed out into the oral cavity ${ }^{52}$. Its proteome and peptidome has been already investigated ${ }^{52-54}$. This fluid could be useful for the discovery of novel periodontal disease markers ${ }^{55}$.

Nonetheless, the efforts needed to reach the above aims are still demanding. An emblematic example is represented by various proteomic studies performed to find salivary biomarkers of OSCC. It is surprising that many of these studies showed little overlap. The only potential biomarkers common to several proteomic studies on OSCC were ATT and some components of the complement, which should be largely investigated to establish their sensitivity, specificity, accuracy and positive predictive value. As discussed in the previous sections, since different proteomic platforms cover different proteomes, it is not surprising that different proteomic methodologies identify different biomarkers. However, if the proteomic platforms utilised are similar, the disagreement could partly derive from the low number of patients available in different clinical centers (fortunately). Due to the medical relevance of OSCC, it seems opportune to organise a network of proteomic laboratories to share samples and results of similar pathologies and to organise multicentre research to identify biomarkers characterised by highly significant statistical parameters that can be soon transferred to routine clinical use.

\section{References}

Tabak, LA. A revolution in biomedical assessment: the development of salivary diagnostics. J Dent Educ 2001;65:1335-9.

2 Grassl N, Kulak NA, Pichler G, et al. Ultra-deep and quantitative saliva proteome reveals dynamics of the oral microbiome. Genome Med 2016;8:44.

3 Helmerhorst EJ, Oppenheim FG. Saliva: a dynamic proteome. J Dent Res 2007;86:680-93.

4 Messana I, Inzitari R, Fanali C, et al. Facts and artifacts in proteomics of body fluids. What proteomics of saliva is telling us? J Sep Sci 2008;31:1948-63.

5 Siqueira WL, Salih E, Wan DL, et al. Proteome of human minor salivary gland secretion. J Dent Res 2008;87:445-50.

6 Pisano E, Cabras T, Montaldo C, et al. Peptides of human gingival crevicular fluid determined by HPLC-ESI-MS. Eur J Oral Sci 2005;113:462-8.

7 Inzitari R, Cabras T, Pisano E, et al. HPLC-ESI-MS analysis of oral human fluids reveals that gingival crevicular fluid is the main source of thymosins beta 4 and beta10. J Sep Sci 2009;32:57-63. 
8 Oppenheim FG, Salih E, Siqueira WL, et al. Salivary proteome and its genetic polymorphisms. Ann NY Acad Sci 2007;1098:22-50.

9 Cabras T, Fanali C, Monteiro JA, et al. Tyrosine polysulfation of human salivary histatin 1. A post-translational modification specific of the submandibular gland. J Proteome Res 2007;6:2472-80.

${ }^{10}$ Cabras T, Manconi B, Iavarone F, et al. RP-HPLC-ESI-MS evidenced that salivary cystatin $B$ is detectable in adult human saliva mostly as $S$-modified derivatives: $S$-glutathionyl, S-cysteinyl and S-S 2-mer. J Proteomics 2012;75:908-13.

11 Castagnola M, Inzitari R, Fanali C, et al. The surprising composition of the salivary proteome of preterm human newborn. Mol Cell Proteomics 2011;10:M110.003467.

12 Messana I, Cabras T, Iavarone F, et al. Unraveling the different proteomic platforms. J Sep Sci 2013;36:128-39.

13 Castagnola M, Cabras T, Iavarone F, et al. The human salivary proteome: a critical overview of the results obtained by different proteomic platforms. Expert Rev Proteomics 2012;9:33-46.

14 Quintana M, Palicki O, Lucchi G, et al. Inter-individual variability of protein patterns in saliva of healthy adults. J Proteomics 2009;72:822-30.

15 Al-Tarawneh SK, Border MB, Dibble CF, et al. Defining salivary biomarkers using mass spectrometry-based proteomics: a systematic review. OMICS 2011;15:353-61.

16 Michalski A, Cox J, Mann M. More than 100,000 detectable peptide species elute in single shotgun proteomics runs but the majority is inaccessible to data-dependent LC-MS/MS. J Proteome Res 2011;10:1785-93.

17 Wang Q, Yu Q, Lin Q, et al. Emerging salivary biomarkers by mass spectrometry. Clin Chim Acta 2015;438:214-21.

18 Schafer CA, Schafer JJ, Yakob M, et al. Saliva diagnostics: utilizing oral fluids to determine health status. Monogr Oral Sci 2014;24:88-98.

19 Cuevas-Córdoba B, Santiago-García J. Saliva: a fluid of study for OMICS. OMICS 2014;18:87-97.

${ }_{20} \mathrm{Wu} \mathrm{CC}$, Chu HW, Hsu CW, et al. Saliva proteome profiling reveals potential salivary biomarkers for detection of oral cavity squamous cell carcinoma. Proteomics 2015;15:3394-404.

${ }^{21}$ Hsu CW, Yu JS, Peng PH, et al. Secretome profiling of primary cells reveals that THBS2 is a salivary biomarker of oral cavity squamous cell carcinoma. J Proteome Res 2014;13:4796-807.

22 Jou YJ, Hua CH, Lin CD, et al. S100A8 as potential salivary biomarker of oral squamous cell carcinoma using nanoLCMS/MS. Clin Chim Acta 2014;436:121-9.

23 Jessie K, Jayapalan JJ, Ong KC, et al. Aberrant proteins in the saliva of patients with oral squamous cell carcinoma. Electrophoresis 2013;34:2495-502.

24 Kawahara R, Bollinger JG, Rivera C, et al. A targeted proteomic strategy for the measurement of oral cancer candidate biomarkers in human saliva. Proteomics 2016;16:159-73.

25 Sivadasan P, Gupta MK, Sathe GJ, et al. Human salivary proteome--a resource of potential biomarkers for oral cancer. Proteomics 2015;127(Pt A):89-95.

${ }^{26}$ Krapfenbauer K, Drucker E, Thurnher D. Identification of tumour-related proteins as potential screening markers by proteome analysis-protein profiles of human saliva as a predictive and prognostic tool. EPMA J 2014;5:20.

27 Bonne NJ, Wong DT. Salivary biomarker development using genomic, proteomic and metabolomic approaches. Genome Med 2012;4:82.

28 Wei J, Xie G, Zhou Z, et al. Salivary metabolite signatures of oral cancer and leukoplakia. Int J Cancer 2011;129:2207-17.

29 Wang Q, Gao P, Wang X, et al. The early diagnosis and monitoring of squamous cell carcinoma via saliva metabolomics. Sci Rep 2014;4:6802.

30 Delmonico L, Bravo M, Silvestre RT, et al. Proteomic profile of saliva and plasma from women with impalpable breast lesions. Oncol Lett 2016;12:2145-52.

31 Xiao H, Zhang Y, Kim Y, et al. Differential proteomic analysis of human saliva using tandem mass tags quantification for gastric cancer detection. Sci Rep 2016;6:22165.

32 Camisasca DR, da Rós Gonçalves L, Soares MR, et al. A proteomic approach to compare saliva from individuals with and without oral leukoplakia. J Proteomics 2017;151:43-52.

33 Zhang L, Xiao H, Zhou H, et al. Development of transcriptomic biomarker signature in human saliva to detect lung cancer. Cell Mol Life Sci 2012;69:3341-50.

34 Bassim CW, Ambatipudi KS, Mays JW, et al. Quantitative salivary proteomic differences in oral chronic graft-versushost disease. J Clin Immunol 2012;32:1390-9.

35 Devic I, Shi M, Schubert MM, et al. Proteomic analysis of saliva from patients with oral chronic graft-versus-host disease. Biol Blood Marrow Transplant 2014;20:1048-55.

36 Castagnola M, Picciotti PM, Messana I, et al. Potential applications of human saliva as diagnostic fluid. Acta Otorhinolaryngol Ital 2011;31:347-57.

37 Chen W, Cao H, Lin J, et al. Biomarkers for primary Sjögren's syndrome. Genomics Proteomics Bioinformatics 2015;13:219-23.

38 Katsiougiannis S, Wong DT. The proteomics of saliva in Sjögren's syndrome. Rheum Dis Clin North Am 2016;42:449-56.

39 Bosello S, Peluso G, Iavarone F, et al. Thymosin $\beta 4$ and $\beta 10$ in Sjögren >s syndrome: saliva proteomics and minor salivary glands expression. Arthritis Res Ther 2016;18:229.

40 Kageyama G, Saegusa J, Irino Y, et al. Metabolomics analysis of saliva from patients with primary Sjögren's syndrome. Clin Exp Immunol 2015;182:149-53.

${ }_{41}$ Sanna M, Firinu D, Manconi PE, et al. The salivary proteome profile in patients affected by SAPHO syndrome characterized by a top-down RP-HPLC-ESI-MS platform. Mol Biosyst 2015;11:1552-62.

42 Wormwood KL, Aslebagh R, Channaveerappa D, et al. Salivary proteomics and biomarkers in neurology and psychiatry. Proteomics Clin Appl 2015;9:899-906.

43 Iavarone F, Melis M, Platania G, et al. Characterization of salivary proteins of schizophrenic and bipolar disorder patients by top-down proteomics. J Proteomics 2014;103:15-22.

44 Dominy SS, Brown JN, Ryder MI, et al. Proteomic analysis of saliva in HIV-positive heroin addicts reveals proteins correlated with cognition. PLoS One 2014;9:e89366.

45 Cabras T, Pisano E, Montaldo C, et al. Significant modifi- 
cations of the salivary proteome potentially associated with complications of Down syndrome revealed by top-down proteomics. Mol Cell Proteomics 2013;12:1844-52.

46 Cabras T, Sanna M, Manconi B, et al. Proteomic investigation of whole saliva in Wilson's disease. $\mathrm{J}$ Proteomics 2015;128:154-63.

47 Zhang N, Zhang Z, Feng S, et al. Quantitative analysis of differentially expressed saliva proteins in human immunodeficiency virus type $1(H I V-1)$ infected individuals. Anal Chim Acta 2013;774:61-6.

48 Jagtap P, McGowan T, Bandhakavi S, et al. Deep metaproteomic analysis of human salivary supernatant. Proteomics 2012;12:992-1001.

49 Belstrøm D, Jersie-Christensen RR, Lyon D, et al. Metaproteomics of saliva identifies human protein markers specific for individuals with periodontitis and dental caries compared to orally healthy controls. Peer J 2016;4:e2433.

50 Sun Y, Xia Z, Shang Z, Sun K, et al. Facile preparation of salivary extracellular vesicles for cancer proteomics. Sci Rep 2016;6:24669.
51 Winck FV, Prado Ribeiro AC, Ramos Domingues R, et al. Insights into immune responses in oral cancer through proteomic analysis of saliva and salivary extracellular vesicles. Sci Rep 2015;5:16305.

52 Pisano E, Cabras T, Montaldo C, et al. Peptides of human gingival crevicular fluid determined by HPLC-ESI-MS. Eur J Oral Sci 2005;113:462-8.

53 Bostanci N, Heywood W, Mills K, et al. Application of labelfree absolute quantitative proteomics in human gingival crevicular fluid by LC/MS E (gingival exudatome). J Proteome Res 2010;9:2191-9.

54 Preianò M, Falcone D, Maggisano G, et al. Assessment of pre-analytical and analytical variables affecting peptidome profiling of gingival crevicular fluid by MALDI-TOF mass spectrometry. Clin Chim Acta 2014;437:120-8.

55 Tsuchida S, Satoh M, Kawashima Y, et al. Application of quantitative proteomic analysis using tandem mass tags for discovery and identification of novel biomarkers in periodontal disease. Proteomics 2013;13:2339-50.

Received: September 15, 2016 - Accepted: December 12, 2016

Address for correspondence: Giulio Cesare Passali, Institute of Biochemistry and Clinical Biochemistry, Catholic University, largo F. Vito 1, 00168 Rome, Italy. E-mail: giuliocesare.passali@ unicatt.it 